\title{
The Magnitude of Lift Forces Acting on Drops and Bubbles in Liquids Flowing Inside Microchannels
}

\section{Citation}

Stan, Claudiu A., Audrey K. Ellerbee, Laura Guglielmini, Howard A. Stone, and George M. Whitesides. 2013. The Magnitude of Lift Forces Acting on Drops and Bubbles in Liquids Flowing Inside Microchannels. Lab on a Chip 13, no. 3: 365-376.

\section{Published Version}

doi:10.1039/c2lc41035d

\section{Permanent link}

http://nrs.harvard.edu/urn-3:HUL.InstRepos:11931827

\section{Terms of Use}

This article was downloaded from Harvard University's DASH repository, and is made available under the terms and conditions applicable to Open Access Policy Articles, as set forth at http:// nrs.harvard.edu/urn-3:HUL.InstRepos:dash.current.terms-of-use\#OAP

\section{Share Your Story}

The Harvard community has made this article openly available.

Please share how this access benefits you. Submit a story.

\section{Accessibility}




\title{
The Magnitude of Lift Forces Acting on Drops and Bubbles in Liquids Flowing inside Microchannels
}

\author{
Claudiu A. Stan, ${ }^{1,}$ Audrey K. Ellerbee, ${ }^{2}$ Laura Guglielmini, ${ }^{3}$ \\ Howard A. Stone, ${ }^{4}$ and George M. Whitesides ${ }^{1}$ \\ ${ }^{I}$ Department of Chemistry and Chemical Biology, Harvard University, \\ Cambridge, MA 01238 USA \\ ${ }^{2}$ Department of Electrical Engineering, Stanford University, \\ Stanford, CA 94305 USA \\ ${ }^{3}$ Center for Turbulence Research, Stanford University, Stanford, CA 94305 USA \\ ${ }^{4}$ Department of Mechanical and Aerospace Engineering, Princeton University, \\ Princeton, NJ 08544 USA
}

"corresponding author, e-mail: claudiu.stan@alum.mit.edu 


\section{Abstract}

Hydrodynamic lift forces offer a convenient way to manipulate particles in microfluidic applications, but there is little quantitative information on how non-inertial lift mechanisms act and compete with each other in the confined space of microfluidic channels. This paper reports measurements of lift forces on nearly spherical drops and bubbles, with diameters from one quarter to one half of the width of the channel, flowing in microfluidic channels, under flow conditions characterized by particle capillary numbers $\mathrm{Ca}_{\mathrm{P}}=0.0003-0.3$ and particle Reynolds numbers $\mathrm{Re}_{\mathrm{P}}=0.0001-0.1$. For $\mathrm{Ca}_{\mathrm{P}}<$ 0.01 and $\operatorname{Re}_{\mathrm{P}}<0.01$ the measured lift forces were much larger than predictions of deformation-induced and inertial lift forces found in the literature, probably due to physicochemical hydrodynamic effects at the interface of drops and bubbles, such as the presence of surfactants. The measured forces could be fit with good accuracy using an empirical formula given herein. The empirical formula describes the power-law dependence of the lift force on hydrodynamic parameters (velocity and viscosity of the carrier phase; sizes of channel and drop or bubble), and includes a numerical lift coefficient that depends on the fluids used. The empirical formula using an average lift coefficient of $\sim 500$ predicted, within one order of magnitude, all lift force measurements in channels with cross-sectional dimensions below $1 \mathrm{~mm}$. 


\section{Graphical abstract}

Hydrodynamic lift forces on bubbles and drops in microchannels were measured directly and fitted to an empirical formula.

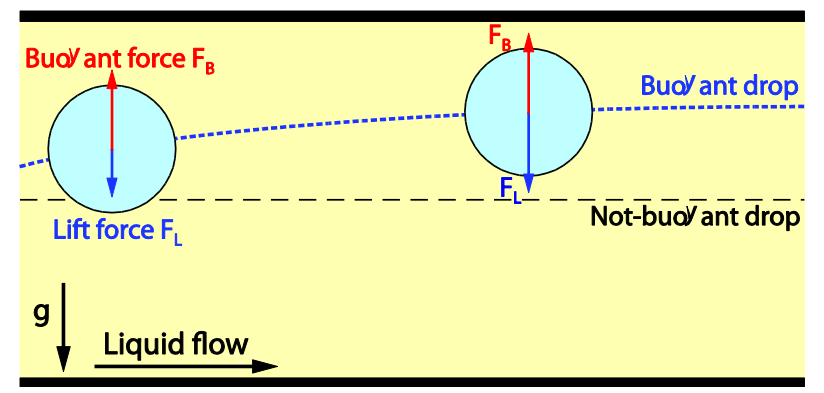




\section{Introduction}

Manipulation of solid particles, liquid drops, gas bubbles, and cells is an important subfield of microfluidics. ${ }^{1-3}$ Immersion in a continuous liquid phase enables the processing, using microfluidic flows, of systems that either cannot flow easily, such as solid particles, or coalesce if brought in contact, such as drops and bubbles. Such twophase microfluidic flows have been used to transport and sort solid particles, ${ }^{1-2,4}$ to manipulate cells, ${ }^{3,5-6}$ and to provide small and independent chemical microreactors. ${ }^{7}$

The methods that have been used to control the motion of dispersed phases in microfluidics can be classified in two categories. The first category uses external force fields (gravitational, electric, magnetic, optic, acoustic) ${ }^{8-13}$ and the second uses forces generated by the flow of the carrier fluid. ${ }^{2,14-15}$

One way in which the flow of a carrier liquid can affect the movement of particles, drops, bubbles, and cells is through the development of lift forces, which refer to hydrodynamic forces that act perpendicular to the direction of the flow. We are interested in one particular case of lift forces: those that act on nearly spherical bubbles and drops that have dimensions smaller than but comparable to the cross-section of the channel, and are carried by a liquid inside microchannels, i.e., channels with crosssectional dimensions smaller than $\sim 1 \mathrm{~mm}$. Such systems are encountered in applications that screen and sort drops at high throughput, ${ }^{16}$ and in applications that require the mechanical isolation of drops from the walls of channels, for example in nucleation studies. ${ }^{17}$

There are several types of lift forces that can act on drops and bubbles: inertial lift forces, ${ }^{14,18-20}$ deformation-induced lift forces, ${ }^{21-22}$ and surfactant ${ }^{23-28}$ and confinement ${ }^{29-31}$ 
effects. The strength and the relative importance of these lift mechanisms depends on the hydrodynamic details of the system under investigation, and identification of the dominant lift mechanism(s) is thus essential for understanding and predicting the effects of lift forces. The inertial and the deformation-induced lift are the best understood lift mechanisms, because it was possibile to design model experiments, involving the flow of small particles or drops in centimeter-sized pipes, in which either inertial ${ }^{19}$ or the deformation-induced lift forces ${ }^{21}$ were dominant, and confinement and surfactant effects were negligible.

Compared to the systems used to study inertial and deformation-induced lift, microfluidic flows are characterized by smaller channel cross-sections and by drops or bubbles that are not small relative to the width of the channel. In microfluidic conditions, surfactant and confinement effects are expected to become more important, ${ }^{32}$ and we reported previously measurements and numerical simulations that indicated that such lift forces contributed to the lift forces in microchannels. ${ }^{33}$ Here we report new experiments in which lift forces that were not inertial, nor were deformation-induced, were dominant; the data acquired in these experiments enabled us to propose simple methods to predict the magnitude of microfluidic lift forces on drops or bubbles, though the origin of the force itself remains unclear.

To quantify the distinctions between microfluidic flows and those in larger pipes and channels, and to characterize our experiments, we used the channel and particle Reynolds numbers $\operatorname{Re}_{\mathrm{C}}$ and $\mathrm{Re}_{\mathrm{P}}$ (eqn (1) and (2), respectively), the particle capillary number $\mathrm{Ca}_{\mathrm{P}}$ (eqn (3)), and the relative size of drops and bubbles, $r / H$, where $r$ is the radius of drop or bubble, and $H$ the relevant cross-sectional dimension of the channel, 
which is the height in our studies. In eqn. (1)-(3), $\rho_{C}$ and $\mu_{C}$ are the density and the viscosity of the continuous phase, $V_{a v g}$ the average fluid velocity in the channel, and $\gamma$ the interfacial surface tension between the fluid particle and the continuous phase:

$$
\begin{aligned}
& \operatorname{Re}_{\mathrm{C}}=\frac{\rho_{C} V_{a v g} H}{\mu_{c}} \\
& \operatorname{Re}_{\mathrm{P}}=\frac{\rho_{C} V_{a v g}}{\mu_{c}} \frac{r^{2}}{H} \\
& \mathrm{Ca}_{\mathrm{P}}=2 \frac{\mu_{c} V_{a v g}}{\gamma} \frac{r}{H}
\end{aligned}
$$

Our prior work used experiments and numerical simulations to explore how lift forces position drops and bubbles transversally in a microchannel The basic results of this work were that (i) the positioning of drops and bubbles can be understood as a result of the balance between inertial lift, deformation-induced lift, and non-hydrodynamic transverse forces such as buoyancy, (ii) analytical theories developed for particles much smaller than the channel dimensions are not as accurate as computational fluid dynamics simulations in predicting the positioning of drops, and (iii) there are discrepancies between simulations and experiments for flow conditions characterized by small particle capillary numbers $\left(\mathrm{Ca}_{\mathrm{P}}<0.01\right)$ and small particle Reynolds numbers $\left(\operatorname{Re}_{\mathrm{P}}<0.01\right)$; these discrepancies might be explained by the contribution of an additional lift mechanism or mechanisms, most likely related to interfacial physicochemical effects ${ }^{32}$ not included in the simulations.

Here we report measurements of the magnitude of lift forces for many combinations of the disperse and continuous phases, with the goals of (i) quantifying the magnitude of lift forces in microchannels, and (ii) predicting the magnitude of these lift 
forces in practical microfluidic applications. We found that for nitrogen bubbles in silicone oil flowing in millimeter-sized channels, the magnitude of the lift forces was approximated well by analytical formulas of deformation-induced lift. In most other cases, however, the lift forces were much larger than the deformation-induced lift. Compared to our previous study, the different research methodology we report heredirect measurement of lift forces in a large number of distinct hydrodynamic conditions - allowed us to (i) identify the conditions under which the additional lift mechanism was the dominant contribution to the overall lift force, and (ii) determine the dependence of the lift force caused by this mechanism on hydrodynamic parameters. Although the magnitude of lift forces caused by the additional mechanism depended on what fluids we used, the large number of distinct measurements we report here (more than 400 measurements, using 21 systems) allowed us to identify common trends in the dependence of lift forces on hydrodynamic parameters.

To date, simple analytical or empirical formulas for the prediction of lift forces exist only for deformation-induced ${ }^{21-22}$ and inertial ${ }^{18,20}$ lift forces. The lift forces we measured were up to four orders of magnitude larger than those predicted by these existing formulas. The suprisingly large magnitude of the lift forces we observed is practically important for microfluidic applications, because it implies that lift forces can be used to manipulate drops and bubbles more effectively than could have been envisioned previously.

Motivated by the discrepancy between our measurements and existing hydrodynamic lift models, and by the lack of a simple way to predict the lift forces acting on finite-sized drops and bubbles in microchannels, we developed an empirical formula, 
eqn (4), that fits the magnitude of the measured lift force for flow conditions characterized by $\mathrm{Ca}_{\mathrm{P}}<0.01$ and $\mathrm{Re}_{\mathrm{P}}<0.01$,

$$
F_{L, \text { empirical }}=C_{L} \mu_{c} V_{\text {avg }} r\left(\frac{r}{H}\right)^{3}\left(\frac{d}{H}\right)
$$

In eqn (4), $F_{L, \text { empirical }}$ is the lift force directed towards the center of the microchannel and $d$ the distance between the drop and the center of the channel; eqn (4) is applicable near the center of the channel, region that we defined here by $d \leq 0.15 \mathrm{H}$, and implies that the drops or bubbles are (nearly) spherical, because $\mathrm{Ca}_{\mathrm{P}}<0.01 .{ }^{34} C_{L}$ is a numerical lift coefficient characteristic of the pair of carrier and drop or bubble fluids, and varied between approximately 100 and 1300 for the systems we surveyed. All variables and parameters in our paper are expressed in SI units.

\section{Background}

The motions of drops and bubbles in microchannels are influenced by several types of hydrodynamic lift forces. Two of these types are particularly relevant to our investigation: the deformation-induced lift force $\mathrm{e}^{21-22}$ and the inertial lift force. ${ }^{14,18,20}$ The deformation-induced lift force pushes drops and bubbles towards the center of the microchannels for drops and bubbles much less viscous than the continuous fluid (the case of our experiments); more specifically, Chan and Leal ${ }^{22}$ give the condition $\kappa>10$ for a lift force directed towards the center of the channel, where $\kappa=\mu_{\text {drop }} / \mu_{C}$ is the ratio of viscosities of the drop or bubble, $\mu_{d r o p}$, and of the carrier fluid, $\mu_{C}$. The inertial lift force has the opposite effect in the central region of the microchannel, pushing drops and bubbles away from the center. The magnitude of the deformation-induced lift force can 
be predicted using the analytical result of Chan and Leal ${ }^{22}$ (eqn (5)-(6)). Eqn (5) is accurate as long as the drop or bubble is not too close to the walls, e.g. the separation between the drop and the wall is larger than the diameter of the drop or bubble.

$$
\begin{gathered}
F_{L, \text { deformation }}=\mathrm{Ca}_{\mathrm{P}} \mu_{c} V_{\text {avg }} r\left(\frac{r}{H}\right)^{2}\left(\frac{d}{H}\right) f(\kappa) \\
f(\kappa)=\frac{72 \pi}{(\kappa+1)^{3}}\left(\frac{11 \kappa+10}{105}\left(3 \kappa^{2}-\kappa+8\right)-\frac{(19 \kappa+16)}{42(3 \kappa+2)(\kappa+4)}\left(24 \kappa^{3}+73 \kappa^{2}+36 \kappa-13\right)\right)(6)
\end{gathered}
$$

We used the experimental measurements of the inertial lift force in microchannels made by Di Carlo et al. ${ }^{18}$ to extract an equation that fits the inertial lift force, $F_{L, \text { inertial }}^{\text {center }}$, (eqn (7)) in the region near the center of the channel where $d / H<0.1$. The sign of $F_{L, \text { inertial }}^{c e n t}$ is negative, because the inertial lift pushes particles away from the center of the channel.

$$
F_{L, \text { inertial }}^{\text {center }}=-10 \operatorname{Re}_{\mathrm{P}} \mu_{c} V_{\text {avg }} r\left(\frac{d}{H}\right)
$$

We arranged the terms of eqn (4), (5) and (7) similarly to illustrate their common features; all these formulas contain a term with the dimension of force, $\mu_{C} V_{a v g} r$, a dependence on the relative size and position of the bubbles or drops, $r / H$ and $d / H$, and a numerical factor that is characteristic of the lift mechanism - for example, the capillary number in the case of deformation-induced lift.

\section{Experimental design}

\section{Experimental apparatus}

The experimental apparatus is the same as that we used in our previous study ${ }^{33}$ (Fig. 1;

Fig. 1(a) and Fig. 1(b) in this paper are modified versions of Fig. 2(a) and Fig 1(a) from 
that work). Bubbles or drops with radii $r$ ranging from $H / 8$ to $H / 4$, produced using a flowfocusing nozzle, flowed in a continuous liquid phase inside a horizontal microchannel whose temperature was controlled to vary the size of drops and bubbles, ${ }^{35}$ and to tune the viscosity of the continuous phase. The drops and bubbles had different densities than the continuous phase, thus they experienced positive or negative buoyancy in the continuous phase; if the flow would stop, drops and bubbles would come in contact with either the top or the bottom of the microchannel. During flow, however, the hydrodynamic lift forces balanced the buoyant force (Fig. 1(b)), and the bubbles and drops obtained a stable transverse position in the microchannel without touching the top or bottom surfaces of the microchannels. We used a microscope and a high-speed camera to record and measure the size and frequency of bubbles and drops, and the distance $d$ between the steady-state transverse position of drops and bubbles and the center of the channel (Fig. 1(b) and 1(c)).

Most experiments reported here were performed in channels with a cross-section of $125(\mathrm{~W}) \times 200(\mathrm{H}) \mu \mathrm{m}$; unless specified otherwise, this channel size was that used for the results reported below. We also performed measurements in channels with cross-sections of $55(\mathrm{~W}) \times 100(\mathrm{H}) \mu \mathrm{m}, 290(\mathrm{~W}) \times 400(\mathrm{H}) \mu \mathrm{m}$, and $1170(\mathrm{~W}) \times 2150(\mathrm{H}) \mu \mathrm{m}$. We used the $1170(\mathrm{~W}) \times 2150(\mathrm{H})-\mu \mathrm{m}$ channel (a "millifluidic", rather than microfluidic channel) to search for differences in effects of hydrodynamic lift between microfluidic flow conditions and flow in larger, millimeter- and centimeter-sized, channels and pipes. Because in larger channels the deformation-induced and inertial lift forces caused phenomena, such as the transverse migration of drops or bubbles, that were predicted quantitatively using analytical predictions of the deformation-induced and inertial lift 
forces, ${ }^{21-22,36}$ we expected that these analytical predictions would be sufficient to explain quantitatively the phenomenon of supporting buoyant drops and bubbles as well. If this were the case, quantitative differences between how buoyant drops or bubbles were "levitated" by hydrodynamic lift in microchannels and in "millichannels" would show that in microchannels the dominant lift force mechanism could be different than in larger channels.

We chose the pairs of dispersed and continuous phase fluids that we investigated according to the following criteria: (i) they were relatively common and commonly used in microfluidic applications, (ii) they were chemically compatible with the materials of the microfluidic channels, e.g. polydimethylsiloxane (PDMS), poly(methyl methacrylate) (PMMA), glass, (iii) they had viscosities larger than $\sim 5 \mathrm{mPa} \cdot \mathrm{s}$, because for the flow conditions we used, bubbles and drops often did not experience a centering lift force in carriers with lower viscosities, and (iv) the difference in densities between the continuous and dispersed phases were on the order of $1 \mathrm{~g} / \mathrm{cm}^{3}$, because the changes in the steadystate position caused by lower density differences were too small to resolve optically. The continuous phase fluids used in our study were perfluoroperhydrophenanthrene (PFPHP) and perfluoromethyldecalin (PFMD), pure or mixed with variable amounts of the surfactant 1H,1H,2H,2H-perfluorooctanol (THPFO); silicone oil (RT500); and Dynalene SF (DySF, a mixture of alkylated aromatic hydrocarbons designed for use in heat exchangers). Table ST1 in the ESI† contains further information about the fluids we used. 
Fig. 1 Experimental setup. Buoyant bubbles or drops flow in a tilted but axially horizontal microfluidic channel and experience hydrodynamic lift forces that balance their buoyancy. a) Experimental setup: drops or bubbles, flowing in continuous phase within a transparent horizontal microfluidic channel, were observed using a microscope. The bubbles or drops were produced by a flow-focusing nozzle, and the temperature of the channel could be varied between -20 and $60{ }^{\circ} \mathrm{C}$. b) The lift force balanced the buoyant force when the drop or bubble traveled at a steady state distance $d$ from the center of the channel; $d$ was measured from recorded images of the channel. c) Tilting the setup in the plane of the cross-section of the channel reduced the magnitude of the components of buoyant and lift forces perpendicular to the imaging axis, and allowed the investigation of smaller lift forces.

a)

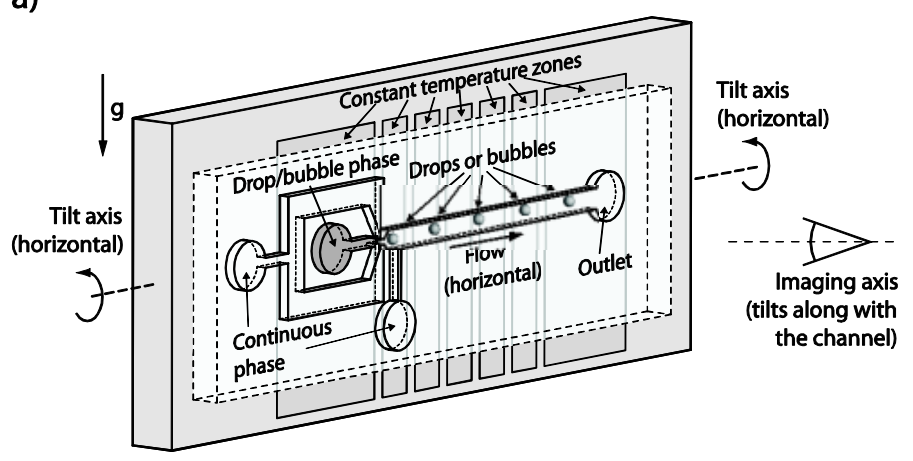

b)

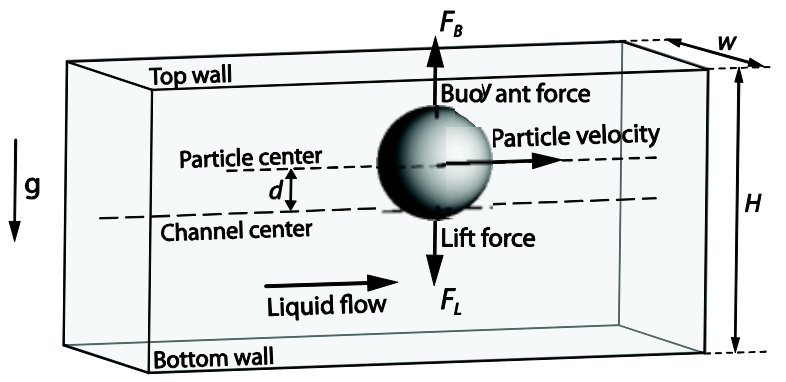

c)

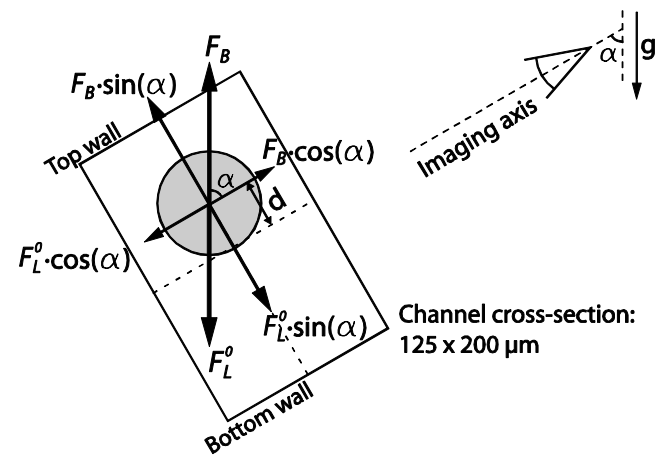




\section{The measurement of hydrodynamic lift forces}

When drops and bubbles reach their equilibrium transverse position, the buoyant force is balanced by the lift force. We measured the lift force at the steady state by equating it with the buoyant force (Fig. 1(b)). Our method is not commonly used in experimental studies of hydrodynamic lift. In contrast, because

buoyant forces are usually large relative to lift forces, the lift forces were calculated in other studies using the viscous drift velocity of neutrally-buoyant particles. ${ }^{21-22,26}$

For a given pair of continuous and dispersed phase liquids and a given drop size, only one measurement of the lift force is possible for the channel orientation shown in Fig. 1(a), because the buoyant force $F_{B}$ has a unique value. To tune the magnitude of the buoyant force in order to investigate a wider range of magnitudes of lift forces, we used the method shown in Fig. 1(c): we tilted the whole experimental setup while keeping the direction of flow horizontal. Because the imaging axis remained in the same orientation relative to the cross section of the channel, we measured $d$ along the longer side of the cross section; $d$ is thus the deviation due to a lift force equal to the component of the buoyant force that is perpendicular to the imaging axis $\left(F_{L}=F_{B} \sin (\alpha)\right.$, where $\alpha$ is the tilt angle). Varying $\alpha$ between $90^{\circ}$ (the channel orientation shown in Fig. 1(a)) and $0^{\circ}$, we could investigate lift forces whose magnitude varied from $F_{B}$ to zero.

Tilting the channel to investigate different magnitudes of the lift force is an approximate method, because if $\alpha$ is below $90^{\circ}$ the drop acquires a deviation along the imaging axis as well; this deviation makes the drop position, and the hydrodynamic forces it experiences, different from the those of drops less buoyant by a factor of $\sin (\alpha)$ and flowing in a channel oriented at $90^{\circ}$. To evaluate how much the drop would deviate 
along the imaging axis, we can use eqn (4) and replace $\mathrm{H}$ with $\mathrm{W}$. The drop deviation due to the same buoyant force is smaller along the imaging axis than perpendicular to it, by a factor of $(\mathrm{H} / \mathrm{W})^{4}$. For our channel cross-sections, which were approximately twice taller than wide, the factor should be close to 16; experimentally, we observed a factor on the order of 10 in the case of the $125(\mathrm{~W}) \times 200(\mathrm{H})-\mu \mathrm{m}$ channel. This factor implies than even when we used the tilting method to investigate weak lift forces that were barely sufficient to prevent the drop from touching the top or bottom of the channel at $\alpha=90^{\circ}$, the "sideways" displacement of the drop was too small to make the drop contact the side walls at $\alpha=0^{\circ}$.

Because it induces a deviation along the imaging axis, the tilting method is useful only when this deviation is relatively small, which is the case when the width of the channel is significantly shorter than its height; we recommend its use only in channels whose height is at least 1.5 times larger than their width. We expect tilting to introduce a small systematic error of underestimating the magnitude of lift forces, because a drop displaced towards the side walls experiences a smaller difference in the range of shear rates across its surface than a drop on the centerline; we did not apply corrections to the data, however, because we could not quantify this systematic error.

\section{The measurement of the dependence of lift forces on hydrodynamic parameters}

To characterize quantitatively the strength of the lift forces, we varied the hydrodynamic parameters of the system and we measured $F_{L}$ and $d$.

In eqn (5) and (7), the lift forces depend on $d$ linearly. The deformation-induced, and the inertial lift forces in microchannels are therefore analogous to a spring; 
depending on which one of the two mechanisms dominate, the "hydrodynamic spring" either restores or repels the drops and bubbles from the center of the channel. All measurements reported here were taken in the regime in which the drops and bubbles experienced an overall lift force that pushed them towards the center; the inertial lift force was therefore weaker than the combined effect of lift forces that acted to center drops and bubbles.

If the lift force depends on the distance of drops and bubbles from the center of the channel, and increases the further from the center the drop or bubble is, the balance between lift and buoyancy is achieved when the drop or bubble is at a steady distance $d$ from the center (Fig. 1(b)). Our principle for measuring the lift force is similar to the measurement of the spring constant of a spring by hanging test weights and measuring the elongation of the spring: the lift corresponds to the spring, the buoyant force corresponds to the weight of the test mass, and $d$ to the elongation. The "spring constant" $F_{L} / d$ of the lift force depends on the hydrodynamic parameters of the system, which here are the viscosities, densities, and flow rates of both the carrier liquid and of the dispersed phase, the surface tension, and the size of the channel and of drops.

\section{Results}

\section{The dependence of lift forces on the distance from the center of the channel}

We investigated first our hypothesis that the lift force acts as an effective spring to restore drops and bubbles to the center of the channel, and that the dependence of the lift force on the distance from the center of the channel is approximately linear in $d / H$, as expressed in eqn (4). Fig. 2(a) shows how the lift force on water drops (diameter $\sim 160$ 
$\mu \mathrm{m})$ flowing in PFPHP with 5\% (v/v) THPFO depends on $d / H$ for a channel with a rectangular cross-section of $290(\mathrm{~W}) \times 400(\mathrm{H}) \mu \mathrm{m}$. The distance from the center was measured along the $400 \mu \mathrm{m}$ dimension (i.e., the height) of the channel, as shown in Fig. 1(c). We set the tilting angle $\alpha$ at several values between $0^{\circ}$ and $90^{\circ}$ to record the data shown in Fig. 2(a).

The combination of fluids we chose for this measurement—water as the dispersed phase, and a fluorocarbon liquid (PFPHP) containing water-insoluble surfactant (THPFO) as the continuous phase-is typical for microfluidic applications in which drops are used as chemical microreactors. ${ }^{7}$ For the data set presented in Fig. 2(a), the lift force increases monotonically with the distance from the center, and the dependence between the lift force and $d$ is approximately linear from the center of the channel to approximately $d=$ $0.15 \mathrm{H}$. The linear dependence of the lift force on $d$ near the center of the channel does not depend on the geometry of microchannels. Fig. 2(b) shows the same measurements performed with smaller drops (diameter $\sim 75 \mu \mathrm{m}$ ) in a smaller channel $(125(\mathrm{~W}) \times 200(\mathrm{H})$ $\mu \mathrm{m})$; although the uncertainty of individual measurements is larger in smaller channels, the results are consistent with a linear dependence. Similar measurements we have made for other pairs of fluids have also shown that the lift force increases linearly with $d$ up to approximately $d=0.15 \mathrm{H}$. The nonlinear increase in the lift force as the bubble approaches the wall was most likely caused by the confinement of the flow of the continuous phase between the bubble and the wall. For example, wall-induced lift effects have been reported previously in the literature. ${ }^{29-31}$ 
Fig. 2 Dependence of the lift force on bubbles of water in a fluorocarbon liquid mixture (PFPHP $+5 \%$ THPFO) on their distance $d$ from the center of the channel. The distance was normalized to the height of the channel, $H$. The symbols represent experimental measurements, the lines are linear fits of data with $d / H \leq 0.15$, and the error bars reflect the uncertainty of measurements. a) Lift force on drops of water in a microchannel channel with a cross-section of $290(\mathrm{~W}) \times 400(\mathrm{H})$ $\mu \mathrm{m}$. b) Data for smaller drops and the same liquids in a smaller channel $(125(\mathrm{~W}) \times 200(\mathrm{H}) \mu \mathrm{m})$ at two different flow velocities; the lift force increased faster with $d$ when the flow velocity was larger.
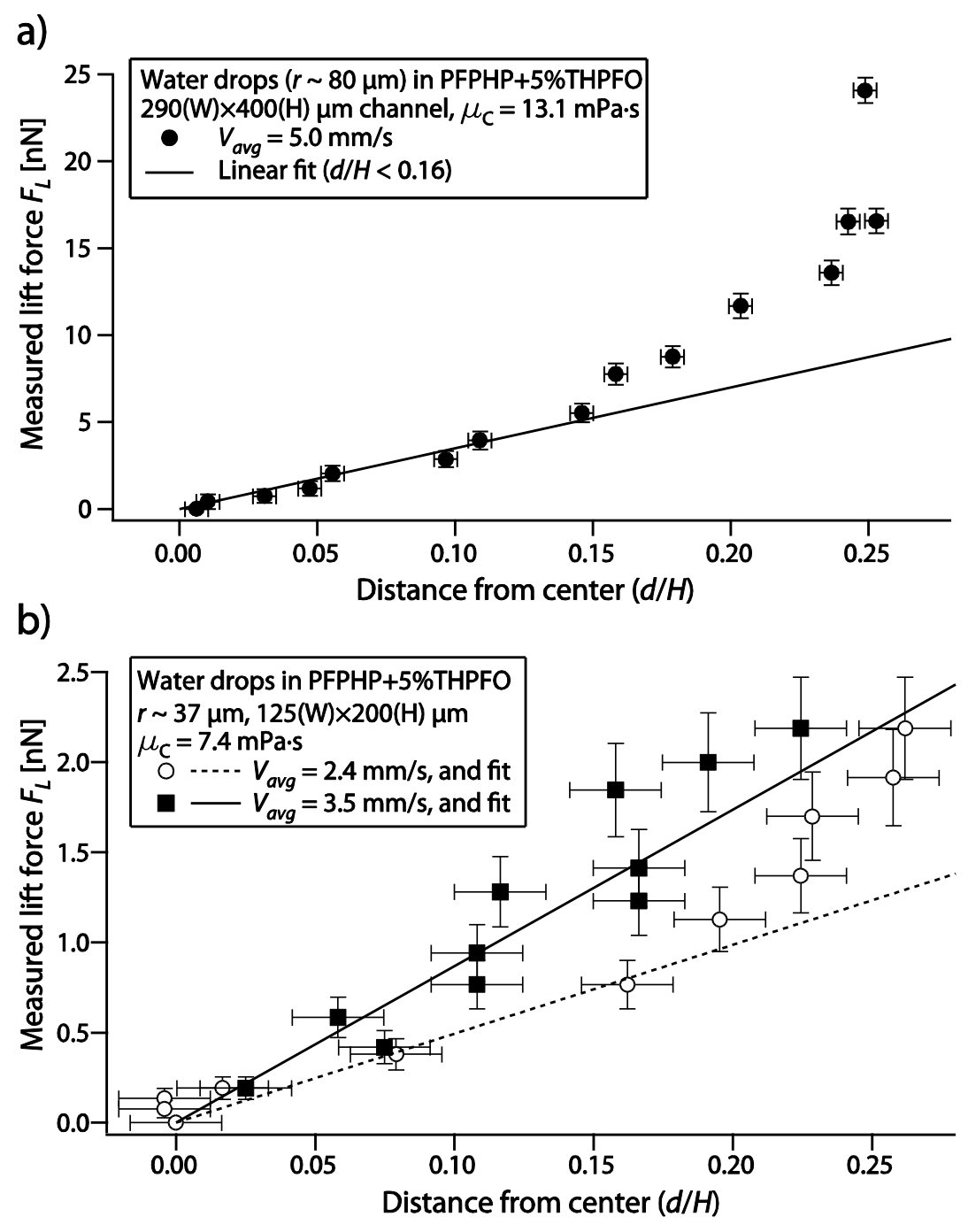
The two data sets in Fig. 2(b) illustrate that the lift force depends on hydrodynamic parameters: an increase in the velocity of the continuous phase increases the slope of the dependency of the lift force on $d$. These two data sets also suggest how to compare properly the lift forces for different hydrodynamic conditions: due to the linear relationship between $F_{L}$ and $d$, the "strength of the lift force" is determined by the coefficient of proportionality between $F_{L}$ and $d$-the "spring constant" in our analogy.

\section{Comparisons between measured lift forces and analytical predictions}

Hydrodynamic lift forces can depend in a complicated manner on many of the hydrodynamic properties of the system, i.e., fluids used, channel design, and fluid hydrodynamic properties. A careful empirical analysis of lift forces must take into account all hydrodynamic parameters of the system, but we can start to understand the lift phenomena by comparing measured lift forces with analytical predictions. We compared the experimentally measured forces with the lift forces predicted to occur through deformation (eqn (5)) and inertial (eqn (7)) mechanisms. Fig. 3 shows the ratio between the measured lift force and the predicted deformation-induced (Fig. 3(a)) and inertial (Fig. 3(b)) lift forces. We grouped our measurements from more than 400 distinct experiments into two data sets. The first set contains the experiments in the large "millichannel" using nitrogen bubbles in silicone oil. The second set groups 20 subsets of experiments, each subset containing data for a different pair of disperse and continuous phases; all these experimental subsets of data have been acquired in microfluidic channels, i.e., channels with cross-sectional dimensions below $1 \mathrm{~mm}$. We plotted the microfluidic data subsets together to illustrate general trends in the data; a detailed list 
with the hydrodynamic parameters and the measured lift forces for all experiments can be found in the ESI $\uparrow$.

The noticeable vertical spread of the second data set in Fig. 3 has two major sources. One is the variation of the lift force due to using different pairs of fluids, variation which is accounted in eqn (4) by the empirical coefficient $C_{L}$. The second source is the normalization of the lift force to the deformation-induced lift force described by eqn (5) which assumes dependencies on hydrodynamic parameters, such as the linear dependence of $F_{L}$ on $d$, that are approximate. To reduce force measurment errors when $d$ was comparable to the optical resolution of our imaging setup, we did not use data from experiments in which $-2 \mu \mathrm{m}<d<2 \mu \mathrm{m} ; 2 \mu \mathrm{m}$ corresponds to 1.5 or 3 image pixel sizes, depending on the imaging magnification we used in a given experiment.

The second data set shown in Fig. 3 has the characteristics that (i) the measurements followed a common trend, and (ii) the measured lift force was up to 3-4 orders of magnitude larger than both the deformation-induced lift force given by eqn (5) and the inertial lift force given by eqn (7); the largest discrepancies occurred at the smallest values of $\mathrm{Ca}_{\mathrm{P}}$ and $\mathrm{Re}_{\mathrm{P}}$ that we investigated.

The fact that inertial lift forces (Fig. 3(b)) were smaller than the observed lift force was not surprising to us, because we designed our experiments such that inertial effects were negligible, by choosing flow conditions characterized by small channel and particle numbers, $\operatorname{Re}_{\mathrm{C}}<1$ and $\operatorname{Re}_{\mathrm{P}}<1$. Under the conditions typical in our experimentsmicrofluidic channels, continuous phase fluids with viscosities 10-100 times larger than water, and average flow velocities less than $0.1 \mathrm{~m} / \mathrm{s}$ - inertial lift forces are much smaller 
than those encountered in microfluidic s sorting applications based on inertial effects. ${ }^{15}$ Fig. 3(b) illustrates that the inertial lift made a negligible contribution to the total lift force.

The measured lift forces were larger than deformation-induced lift forces for both data sets (Fig. 3(a)). The prediction for the deformation-induced lift force was based on eqn (5), which was developed for small (relative to the size of the channel) bubbles or drops flowing in a circular pipe. In our experiments the bubbles or drops were larger $(H / 8$ $<r<H / 4$ ), and we expected the actual deformation-induced lift force to be larger than what eqn (5) predicts, because of confinement effects. ${ }^{29-31}$ In a previous study, ${ }^{33}$ we used numerical simulations to evaluate the contribution of confinement effects on the deformation-induced lift force. For the experiments with nitrogen bubbles in silicone oil (see Fig. 8 in ref. 31), simulations predicted accurately the position of bubbles, indicating that numerical simulations evaluated accurately the effect of confinement on the magnitude of the deformation-induced lift force: the actual deformation-induced lift force was larger by a factor of 2.4 than the prediction of eqn (5).

The data collected in microfluidic channels was larger by up to three orders of magnitude than the analytical prediction. We believe that such a large factor cannot be explained by confinement effects because the relative sizes $r / H$ of drops and bubbles, which quantify the degree of confinement, varied only within a factor of 2 among our measurements. The large lift forces observed at low capillary numbers were probably caused by a different lift mechanism, whose effects became dominant as the size of the channel is reduced. 
Fig. 3 Comparisons between measured lift forces and analytical predictions. The data points represent the ratio of measurements to predictions of the magnitude of lift forces. Approximately 400 independent measurements used different continuous and dispersed phase fluids, channel and drop or bubble sizes, and hydrodynamic parameters. We separated the data into two sets, depending on the size of the channels; the microfluidic data set is composed of multiple subsets of data from 20 different pairs of fluids. The error bars represent the uncertainty of measurements; for clarity, only a few are shown. a) Comparison with an analytical model of the deformation-induced lift force. b) Comparison with an empirical model of the inertial lift force.

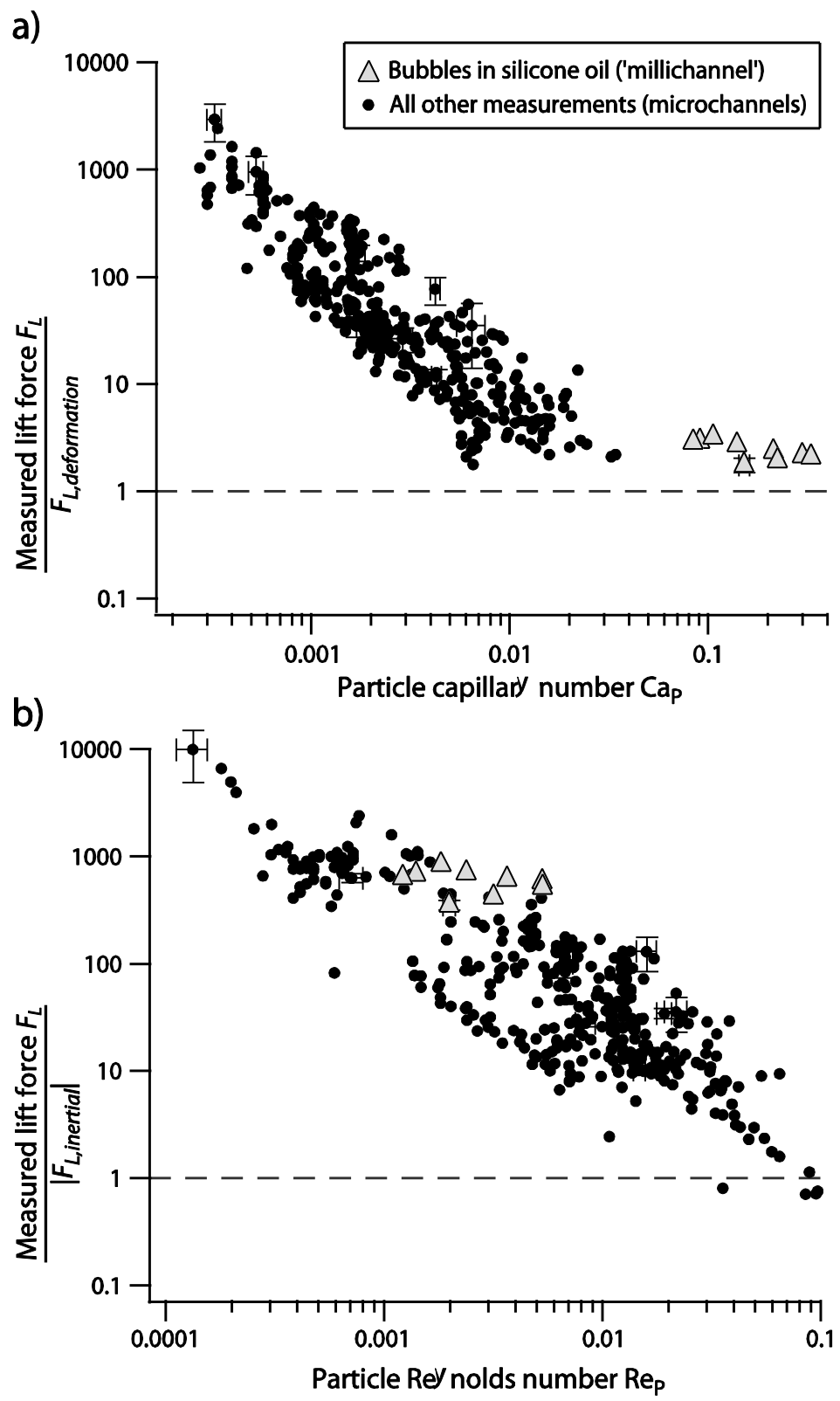




\section{An empirical formula for the prediction of lift forces in microchannels}

Fig. 3 shows that the lift forces observed at low capillary numbers are much larger than those generated by inertial and deformation effects, and might thus be explained instead by confinement or by surfactant effects. For the experiments shown in Fig. 3, none of the continuous phase fluids was chemically pure (i.e., composed of a single molecular species), and many contained actual surfactants. In numerical simulations, surfactants have been shown to lead to the centering of drops in Poiseuille flows; ${ }^{25-26,28}$ this centering phenomenon indicates the presence of a lift force mechanism associated with the presence of surfactants.

Past work on numerical simulations of drop or bubble transport inside channels in

the presence of surfactants ${ }^{25-26,28}$ did not include easy-to-use formulas for the calculation of lift forces, and to date we could not derive an analytical formula for lift forces caused by surfactants. The lift forces that we measured, however, showed a consistent trend in their dependence on $\mathrm{Ca}_{\mathrm{P}}\left(\right.$ Fig. 3(a)) and $\operatorname{Re}_{\mathrm{P}}\left(\right.$ Fig. 3(b)) $: F_{L} / F_{L, \text { deformation }}$ and $F_{L} /\left|F_{L, \text { inertial }}\right|$ are approximately inversely proportional to $\mathrm{Ca}_{\mathrm{P}}$ and $\mathrm{Re}_{\mathrm{P}}$, respectively. These trends suggested that the actual lift force can be fitted by a formula that is similar in form the formulas for the deformation-induced (eqn (5)) and inertial lift forces (eqn (7)), but does not contain $\mathrm{Ca}_{\mathrm{P}}$ or $\mathrm{Re}_{\mathrm{P}}$.

In our search for an empirical formula, we assumed that the actual lift force has a power-law dependence on the hydrodynamic parameters of the system, just as do the inertial and deformation-induced lift forces, and that the form of the equation of the lift force is similar to eqn (5) and (7). Eqn (8) shows such a formula for the lift force, including the power-law exponents $x, y$ and $t$. 


$$
F_{L, \text { empirical }}=C_{L}\left(\mu_{c} V_{\text {avg }} r\right) \frac{r^{x} d^{y}}{H^{t}}
$$

In eqn (8), $C_{L}$ is a numerical lift coefficient, the combination of parameters $\mu_{C} V_{a v g} r$ has the dimensions of force, and the exponents $x, y$, and $t$ must satisfy the condition $x+y=t$ to assure the correct dimensionality of the formula. Eqn (8) is quite general, and it also describes the deformation-induced lift force (eqn (5)) when $x=2, y=$ 1, $t=3$, and $C_{L}=f(\kappa) \mathrm{Ca}$, and the inertial lift force (eqn (7)) when $x=0, y=1, t=1$, and $C_{L}=-10 \mathrm{Re}_{\mathrm{P}}$

We determined the exponents $x, y$ and $t$ by fitting our measurements. Fig. 2 shows that the lift force depends linearly on $d / H$ near the center of the channel. To provide a simple empirical formula, we decided to restrict the formula's applicability to $d / H \leq 0.15$, where the lift force depends linearly on $d / H$ and $y=1$; most of the measurements shown in Fig. 3 were performed in the "linear" regime of $d / H \leq 0.15$.

To determine the dependence of the lift force on $r$, we performed measurements in which we kept all hydrodynamic parameters, except $r$ and $d$, constant. To take into account the variation of $d$ within a given data set, we divided the lift force by $d$ and we plotted the "spring constant" as a function of $r$. Fig. 4(a) shows three sets of such experiments for different pairs of fluids. The measurements were consistent with a power-law dependence, with an exponent between 3.59 and 4.67, and we chose the integer value 4 for the overall exponent of $r$. The overall exponent includes an additional unit of power from the $\mu_{C} V_{a v g} r$ factor, therefore $x=3$, and $t=x+y=4$. 
Fig. 4 Power-law dependencies of the measured lift forces. a) The lift force, normalized by its dependence on $d$, is proportional to approximately the fourth power of the radius of drops or bubbles. b) The lift force, normalized by its dependence on $d$ and $r$, is proportional to approximately the first power of the product of viscosity and velocity of the continuous phase. The error bars represent the uncertainty of measurements; for clarity, only a few are shown.
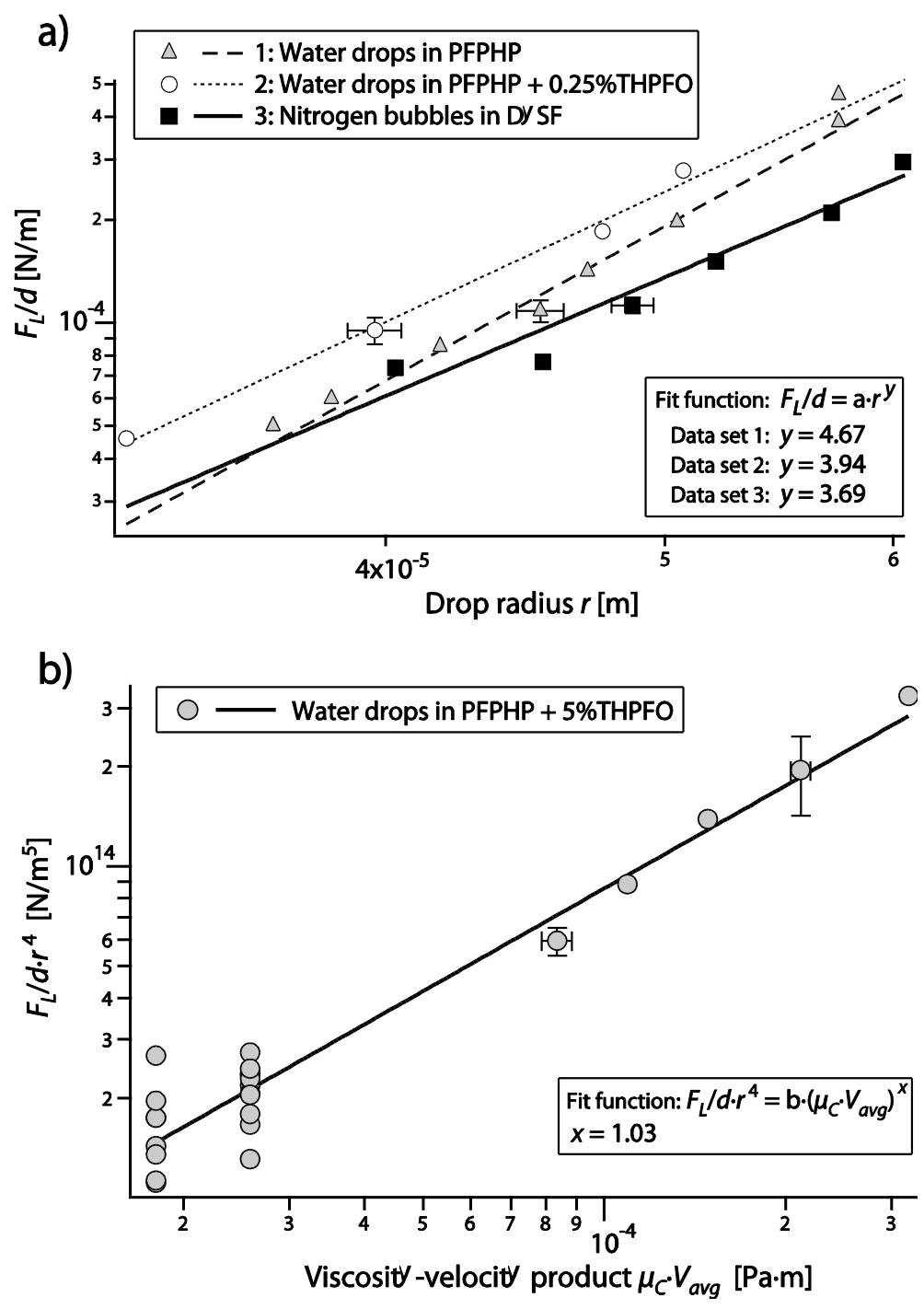
In eqn (8), the explicit dependence of the lift force on the product $\mu_{C} V_{\text {avg }}$ is linear, but the overall dependence could have a different power-law exponent because $\mu_{C}$, or $V_{a v g}$, or both, can be part of an non-dimensional combination of parameters included in $C_{L}$; for example, in the case of the deformation-induced lift force (eqn (5)) the overall exponent of $\mu_{C} V_{\text {avg }}$ is 2 because of the additional $\mu_{C} V_{\text {avg }}$ term included in the capillary number $\mathrm{Ca}_{\mathrm{P}}$ (eqn (3)). To determine the overall exponent under which $\mu_{C} V_{\text {avg }}$ enters into the empirical formula, we fitted the lift force as a power law function of $\mu_{C} V_{\text {avg. }}$. Ideally, such an analysis should be carried on a data set in which only $\mu_{C}, V_{\text {avg }}$, and $d$ vary. In our experiments it was not always possible to keep the size of drops or bubbles constant; we have thus normalized the lift force not only by $d$ but also by its dependence on $r$. Fig. 4(b) shows the dependence of $F_{L} / d \cdot r^{4}$ on $\mu_{C} V_{\text {avg }}$, for water drops in PFPHP $+5 \%$ THPFO, in a channel with a cross section of $125(\mathrm{~W}) \times 200(\mathrm{H}) \mu \mathrm{m}$. The exponent determined by fitting is 1.03 , which was close to 1 and indicated that there was no additional dependence on $\mu_{C} V_{\text {avg }}$ "hidden" in the lift coefficient $C_{L}$.

We performed a similar analysis for other pairs of fluids. In most cases the overall exponent of $\mu_{C} V_{\text {avg }}$ had a value close to 1 , but in a few cases the exponent was closer to 0.5 or to 2. Different values of the overall exponent of $\mu_{C} V_{\text {avg }}$ can be a signature of different lift mechanisms, therefore the range of exponents that we obtained by fitting could indicate that we observed several different lift mechanisms. Since the goal of our present work was to derive a practical empirical formula rather than to categorize in detail all lift mechanisms, we chose to use an overall exponent of 1 in the empirical formula. This choice fitted with good accuracy most of our measurements. Out of the cases in which the exponent was not close to 1, we distinguished those with an overall 
exponent of 2 because this is the exponent characteristic to the deformation-induced lift force. A linear proportionality between $F_{L}$ and $\left(\mu_{C} V_{a v g}\right)^{2}$ implied that $F_{L}$ was proportional, but not necessarily equal, to the deformation-induced lift force given by eqn (5). We note that most, but not all, measurements on bubbles were best fitted by an overall exponent of 2, while most, but not all, measurements on drops were best fitted by an overall exponent of 1 .

Introducing the exponents $x=3, y=1$, and $\mathrm{t}=4$ into eqn (8) we obtain an empirical equation for the lift force (eqn (9)), which after rearrangement becomes identical to eqn (4).

$$
F_{L, \text { empirical }}=C_{L}\left(\mu_{c} V_{\text {avg }} r\right) \frac{r^{3} d}{H^{4}}
$$

The lift coefficient $C_{L}$ must be determined from experimental measurements for each pair of continuous and dispersed phases. For the data displayed in Fig. 4(b), $C_{L}=$ 1278, and Fig. 5(a) shows the agreement between the prediction of eqn (4) and experimental measurements. Eqn (4) with $C_{L}=1278$ also predicted with good accuracy the lift force acting on drops in a channel with a larger cross-section. Overall, the empirical formula for the lift force provided much better predictions than the predictions of the deformation-induced lift force; the inset of Fig. 5(a) shows the comparison of measurements with the prediction of eqn (5) for the deformation-induced lift force. 
Fig. 5 Comparisons between measurements and the predictions of the empirical (eqn (4)) and deformation-induced (eqn (5)) formulas. a) The lift on drops of water in PFPHP $+5 \%$ THPFO was best predicted by the empirical formula with $C_{L}=1278$. b) The lift on nitrogen bubbles in DySF was best predicted by the deformation-induced formula multiplied with a correction factor of 35. c) All measurements, scaled to the predictions of eqn (4) with $C_{L}=535$.
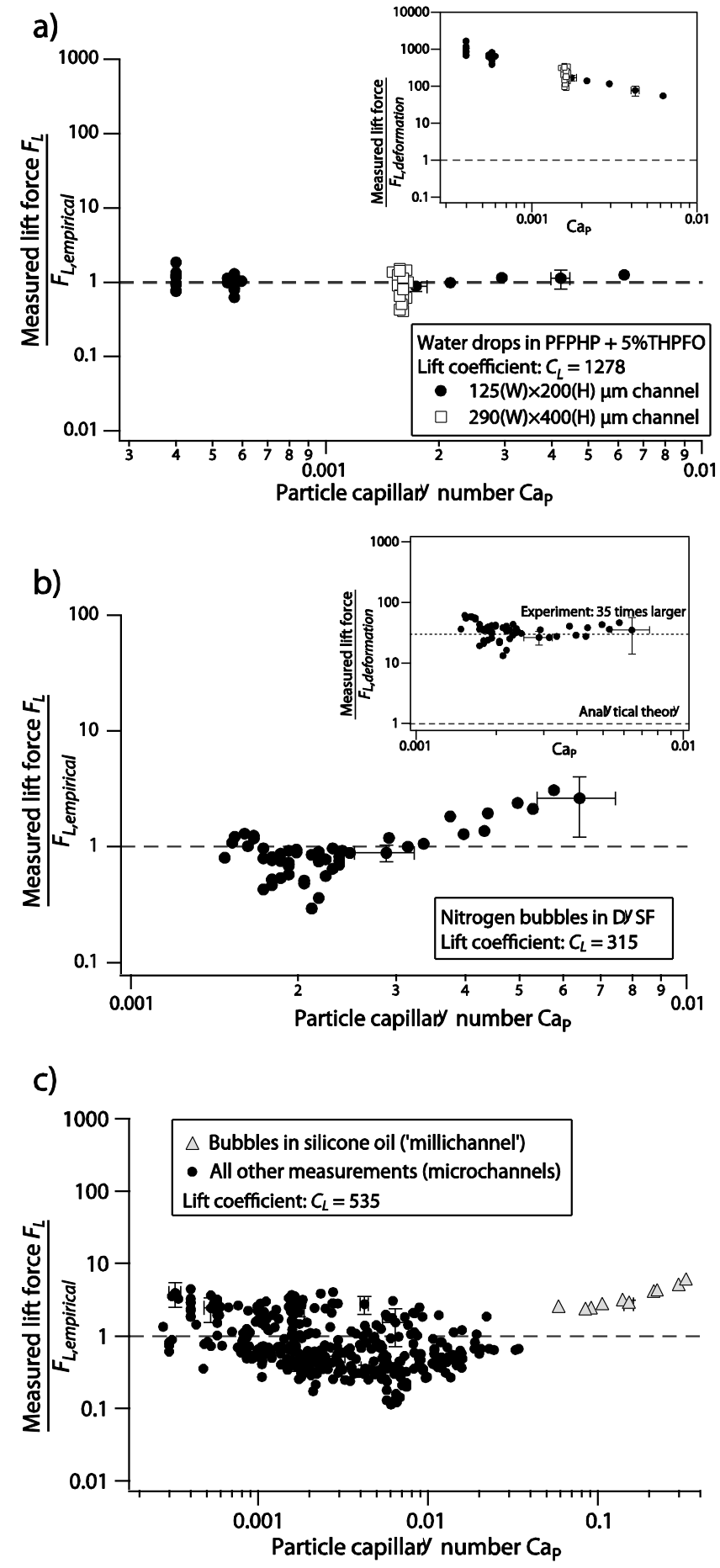
Table 1. Lift force type, lift coefficients $C_{L}$, and correction factors for the deformation lift formula for several combinations of dispersed and continuous phase fluids.

\begin{tabular}{|c|c|c|c|}
\hline System & Lift type & $\begin{array}{l}\text { Lift coefficient } \\
\qquad C_{L}\end{array}$ & $\begin{array}{l}\text { Correction for } \\
\text { deformation lift }\end{array}$ \\
\hline Water drops in PFPHP & Empirical & 261 & - \\
\hline Water drops in PFPHP $+0.25 \%$ PFO & Empirical & 234 & - \\
\hline Water drops in PFPHP $+1 \%$ THPFO & Empirical & 370 & - \\
\hline $\begin{array}{l}\text { Aqueous CsCl solution }(1120-1780 \\
\left.\mathrm{kg} / \mathrm{m}^{3}\right) \text { in PFPHP }+0.1 \% \text { THPFO }\end{array}$ & Empirical & 289 & - \\
\hline Water drops in PFPHP $+5 \%$ THPFO & Empirical & 1278 & - \\
\hline Water drops in THPFO & Empirical & 1026 & - \\
\hline Water drops in PFMD $+2 \%$ THPFO & Empirical & 1250 & - \\
\hline $\begin{array}{c}\text { Aqueous } \mathrm{CsCl} \text { solution }\left(1520 \mathrm{~kg} / \mathrm{m}^{3}\right) \\
\text { in DySF }\end{array}$ & $\begin{array}{l}\text { Proportional to } \\
\text { deformation }\end{array}$ & 581 & 97 \\
\hline Nitrogen bubbles in DySF & $\begin{array}{l}\text { Proportional to } \\
\text { deformation }\end{array}$ & 315 & 35 \\
\hline Nitrogen bubbles in silicone oil & Deformation & - & 2.4 \\
\hline
\end{tabular}


Three combinations of continuous and dispersed phase fluids (nitrogen bubbles in silicone oil; nitrogen bubbles and aqueous $\mathrm{CsCl}$ solutions in $\mathrm{DySF}$ ) exhibited a lift force proportional to $\left(\mu_{C} V_{\text {avg }}\right)^{2}$. In these cases the measured lift force was proportional to the deformation-induced lift force but larger by a constant numerical factor. In one of these cases — nitrogen bubbles in silicone oil—-the correction factor was small (2.4) and can be rationalized as being caused by confinement effects. In microchannel experiments with drops and bubbles in the liquid DySF, the correction factor was much larger. Fig. 5(b) shows the case of nitrogen bubbles in DySF; although the empirical formula (eqn (4)) with $C_{L}=315$ produces good predictions, they are inferior to using the deformationinduced lift formula (eqn (5)) multiplied by a correction factor of 35 .

Table 1 lists the values of $C_{L}$ for a several systems, and a complete list of the 21 pairs of fluids we investigated can be found in Table ST2 in the ESI †. Fig. S1-S5 in the ESI $\dagger$ contain comparisons (such as the ones shown in Fig. 5) between measured and predicted lift forces for all systems. The lift forces were always larger than the deformation-induced lift force, and $C_{L}$ varied between 116 and 1278 among all pairs of liquids that we investigated. Overall, eqn (4), using $C_{L}$ values experimentally determined for each fluid pair investigated, predicted the measured the magnitude of the lift force within a factor of 3 or better, even in cases where the corrected deformation-induced lift force provided better predictions.

We surveyed briefly the effect of the presence of surfactants in the continuous phase in a series of experiments with water drops flowing in a mixture of PFPHP with variable amounts of the surfactant THPFO. The lift coefficients generally became larger as the concentration of THPFO increased, suggesting that the lift force was caused by the 
presence of surfactants. We have not attempted to determine more accurately the dependence of $C_{L}$ on the amount of surfactant because some of the systems in the experimental series, listed in Table ST2 in the ESI†, did not follow the trend of larger $C_{L}$ at higher surfactant concentration.

The necessity to determine experimentally the type of lift force (empirical, or deformation-induced with a correction factor) and the $C_{L}$ complicates the prediction of the lift forces. A less accurate but much simpler prediction can be made using eqn. (4) with an "average" value of $C_{L}$. Fig. 5(c) shows the relation between measured lift forces and those predicted for $C_{L}=535$, which was the average value of $C_{L}$ for the measurements in microfluidic channels. Eqn (4) with $C_{L}=535$ predicts with an accuracy within one order of magnitude all our experimental measurements in microfluidic channels - a significant improvement over using eqn (5) or (7), which can underestimate the lift forces by up to four orders of magnitude.

\section{Discussion}

\section{Distinction from microfluidic studies of inertial lift forces}

The interest in hydrodynamic lift forces acting on particles during channel flow has been recently renewed by demonstrations that used inertial lift forces to order a variety of particles, including live cells, in microfluidic applications. ${ }^{18}$ The microfluidic applications of inertial hydrodynamic effects typically require flow regimes characterized by channel Reynolds numbers $\operatorname{Re}_{\mathrm{C}}>1$, which in practice translates into using carrier fluids with lower viscosities than we investigated, and flows with higher velocities. 
Our investigation addressed a different regime of flow than that used in inertial ordering and sorting applications, and suggests how lift forces can be used to control the motion of drops and bubbles when the continuous phase has viscosities 10-100 times larger than that of water, and flows too slowly to generate significant inertial lift forces. Such an approach has the advantages of requiring smaller amounts of continuous phase (because of the lower rates of flow) and of being able to tune easily the strength of the lift force (by varying $\mu_{C}$ or $V_{a v g}$ ), but is only proven to work on drops and bubbles. The lift forces that we investigated cannot be used to control solid particles; we observed that the lift forces on solid ice particles were much weaker than those on water drops flowing under the same conditions. ${ }^{33}$ Live cells, which are soft objects and can be sorted using deformation-induced lift forces, ${ }^{37-38}$ might however experience lift forces comparable to those predicted by eqn (4) at low capillary numbers.

\section{The investigation of hydrodynamic lift forces in microfluidic conditions}

In our past work, ${ }^{33}$ we employed a research methodology that was distinct from the methodology we used here. There, we investigated the positioning of drops and bubbles due to hydrodynamic lift forces by comparing experimental measurements with numerical simulations of the microfluidic flow. Because numerical simulations required significantly more time than experiments (days or even weeks of CPU time, compared to several minutes for an experiment), the positioning effect of lift forces could only be investigated for a small range of hydrodynamic parameters, and experiments conducted at the lowest $\mathrm{Ca}_{\mathrm{P}}$ and $\mathrm{Re}_{\mathrm{P}}$ that we investigated $\left(\mathrm{Ca}_{\mathrm{P}}<0.001\right.$ and $\left.\mathrm{Re}_{\mathrm{P}}<0.001\right)$ could not be simulated numerically because such simulations require large computational resources. In 
addition, we have not been able to develop a computational approach to incorporate physicochemical effects. Due to these limitations, comparisons of numerical calculations with experimental measurements could only indicate that a lift mechanism that is not inertial nor deformation-based contributed to the lift force.

Here, we took an exclusively experimental approach that allowed us to investigate capillary numbers as low as $\mathrm{Ca}_{\mathrm{P}}=3 \times 10^{-4}$, and we observed very large differences between analytical predictions of inertial and deformation-based lift and our measurements; under these conditions, the lift mechanisms that were not inertial nor deformation-based dominated the lift force. Measurements performed in the regime in which the inertial and the deformation-induced lift force were negligible allowed us to observe a trend in the dependence of the additional lift mechanism on Cap and Re. This trend enabled us to propose an empirical formula to evaluate the lift forces acting on drops and bubbles in microfluidic applications. We believe that the empirical formula, depite its approximate nature, may represent a useful contribution to the fundamental study of hydrodynamic lift—as a starting point and a comparison value for future experimental and numerical studies.

The key features of our experimental approach were (i) to measure the lift forces during steady state flow by balancing them against a known external force (buoyancy) and (ii) to vary the hydrodynamic parameters one at a time and observe their influence on the lift force. Both features were possible because microfluidics allows very good control of experimental parameters; microfluidic techniques offer the possibility to investigate hydrodynamic lift with a higher degree of accuracy than was possible previously. For example, though the change in the direction of the deformation-induced lift force when 
the drops and the carrier fluid have approximately the same viscosities was predicted both analytically ${ }^{22}$ and numerically, ${ }^{39}$ a transverse positioning of drops that was consistent with this phenomenon was not observed experimentally until the microfluidic study of Hur et al. ${ }^{38}$

\section{New effects of hydrodynamic lift forces in microchannels}

During our investigation of lift forces we encountered an apparent paradox. Although all formulas for lift forces caused by different mechanisms, including the empirical formula we proposed, do not depend on the absolute size of the channel, $H$, but on the relative drop size and position, $r / H$ and $d / H$, our experimental observations suggested that the hydrodynamic lift is qualitatively different inside microchannels: (i) we observed the "strongest" lift forces in the smallest channels we used, and (ii) a dependence of the lift force on drops and bubbles such as the one described by our empirical formula was not observed in previous experimental studies that used centimeter- and millimeter-sized channels.

Our results depended on the absolute size of the channel because our measurement technique was not entirely hydrodynamic. Equality between the lift force, which depends on $r / H$, and the buoyant force, which depends on $r$, is only possible for certain values of $H$; these values of $H$ depend on the hydrodynamic properties of the system. For example, we estimated that for the fluids we used in this study, a lift force described by our empirical formula can balance buoyant forces caused by density differences close to $1000 \mathrm{~kg} / \mathrm{m}^{3}$ only if the height of the channel is smaller than a few millimeters. In addition, this balance can be achieved only when the viscosity of the 
continuous phase is larger than a threshold value that increases with the channel size; in the smallest channel we used $(H=100 \mu \mathrm{m})$, but not in larger channels, we could support buoyant drops in continuous phases with viscosities as low as $3 \mathrm{mPa} \cdot \mathrm{s}$. Although the hydrodynamic lift forces do not depend on the absolute size of the channel, they can lead to phenomena that depend on the absolute size of the channel; such phenomena might be observable, and thus practically relevant, only in microchannels.

This type of dependence on the absolute channel size was also the primary reason behind our ability to investigate lift forces over a wide range of particle Renolds and capillary numbers; the average $\mathrm{Ca}$ numbers that we could investigate in a given channel were approximately proportional to the square of the cross section of the channel. Since we used channels whose height varied over one one order of magnitude, variations in the width of the channels account for approximately two of the three orders of magnitude over which we varied $\mathrm{Ca}_{\mathrm{P}}$; the remaining order of magnitude was enabled by combining the techniques of tilting and temperature control.

\section{Interfacial phenomena and the hydrodynamic lift}

A plausible cause for lift forces larger than those generated by the deformation of drops and bubbles is a Marangoni-like interfacial phenomenon at the boundary of drops and bubbles, such as the redistribution of surfactants on a drop or bubble due to the flow of

the continuous phase. ${ }^{24}$ The physical mechanism of the Marangoni effects is: (i) an uneven distribution of surface energy is generated and maintained along the interface of a drop or bubble by conditions such as thermal gradients or uneven shear rates at an interface containing surfactants; (ii) the uneven surface energy causes the flow of the 
interface in the direction of higher surface tension; (iii) the flow of the interface engages the bulk fluids from either side of the interface through viscous friction, a process that can be imagined as the drops "swimming" in the continuous fluid, and is equivalent to the drift of drops or bubbles by due to an applied force; this force is the Marangoni force. Marangoni forces are proportional to the spread of values of the surface tension along the surface of the drop, not to the absolute value of the surface tension; Marangoni forces are thus not directly related to $\mathrm{Ca}_{\mathrm{P}}$, although in an isothermal system they require the presence of a surfactant and depend on its concentration.

The series of experiments in which we used continuous fluids which were mixtures of a base liquid (PFPHP) with variable amounts of surfactant (THPFO) showed that the lift forces were related to the presence of surfactant. Table 1 shows that $C_{L}$, and therefore the lift force, became larger as the concentration of surfactant increased. The increase in the lift force when more surfactant is present is intuitively consistent with a lift mechanism caused by the redistribution of surfactants, but we could not compare quantitatively our measurements with previously reported research. ${ }^{23-26,28}$ Previously reported work on the lift forces caused by surfactants applied either to much larger Reynolds numbers ${ }^{23-24}\left(\operatorname{Re}_{\mathrm{P}} \sim 100\right)$, or to systems with interacting drops or bubbles, ${ }^{23-24,}$

${ }^{27}$ or necessitated the knowledge of dynamic interfacial properties ${ }^{26,28}$ (such as surfactant adsorption and desorption rates) which we could not evaluate for the fluids that we used.

\section{Conclusions}

This paper reports an investigation of the hydrodynamic lift forces acting on drops and bubbles in microchannels. We measured lift forces experimentally, and analyzed these 
measurements to elucidate the mechanisms that cause lift forces, and to provide a simple formula for calculating these lift forces in practical applications. At least two distinct types of mechanisms were responsible for the lift, depending on the combinations of fluids used in the experiment and on the dimensions of the channel.

The first mechanism was the deformation-induced lift force, for which quantitative analytical formulas have been derived previously. We found that for bubbles of nitrogen in silicone oil, flowing in a channel with a millimeter-sized cross-section, the confinement effects made this force larger by a factor of 2.4 than the force predicted by analytical formulas.

In most of the other experiments we observed lift forces up to three orders of magnitude larger than the deformation-induced lift force, while using continuous and dispersed phase fluids that were typical to microfluidic applications. Elucidating the mechanism that causes these surprisingly strong lift forces is an open problem; despite this, we could derive empirically a formula (eqn (4)) that makes the use these lift forces practical in microfluidic applications.

We note that eqn (4) includes an empirical lift factor $C_{L}$ which, for most accurate predictions, has to be determined experimentally for a given pair of carrier liquid and drop or bubble fluids. Alternatively, it is possible to use an "average" value of $C_{L}$ to make order-of-magnitude estimates, and we propose to use eqn (4) with an value $C_{L}=500$ for quick predictions of the magnitude of the lift forces; this prediction agreed, within one order of magnitude, with all measurements we reported here. Our experimental survey of fluids included air bubbles and aqueous drops, and examples from three classes of carrier fluids: fluorocarbon liquids, hydrocarbon liquids, and silicone oils. Among all possible 
combinations of these fluids, it is probable that some systems will not experience a hydrodynamic lift force described by our empirical formula, but we believe that many of them will.

Hydrodynamic lift forces, by themselves, lead to the migration of particles to a few known equilibrium points, such as the center of a channel; in order to achieve further control of the position of particles, external forces must be used. Prediction and control of the position of particles when external forces are present necessitates the knowledge of all forces acting on the particle. Our quantitative investigation of the lift forces, and the empirical formula we proposed, enable the predictive design of microfluidic devices in which the transverse position of drops or bubbles must be precisely controlled.

\section{Acknowledgments}

This work was supported by the US Department of Energy, Division of Materials Sciences \& Engineering, under Award No. DE-FG02-00ER45852. Shared cleanroom facilities funded by the US National Science Foundation under MRSEC award DMR0213805 were utilized to fabricate microfluidic devices.

\section{Notes}

$\dagger$ Electronic Supplementary Information (ESI) available: (1) additional information about the fluids used in this study; (2) a table with the characteristics of the lift forces for all pairs of fluids investigated here; (3) graphs, for each system investigated in microchannels, showing the comparison between experimental measurements and the predictions of deformation-induced and empirical formulae for the lift force; and (4) the hydrodynamic parameters of all experiments. See DOI: 10.1039/b000000x/ 


\section{References}

1. N. Pamme, Lab Chip, 2007, 7, 1644-1659.

2. X. C. Xuan, J. J. Zhu and C. Church, Microfluid. Nanofluid., 2010, 9, 1-16.

3. A. Lenshof and T. Laurell, Chem. Soc. Rev., 2010, 39, 1203-1217.

4. L. R. Huang, E. C. Cox, R. H. Austin and J. C. Sturm, Science, 2004, 304, 987-990.

5. S. K. Sia and G. M. Whitesides, Electrophoresis, 2003, 24, 3563-3576.

6. A. Y. Fu, H. P. Chou, C. Spence, F. H. Arnold and S. R. Quake, Anal. Chem., 2002, 74, 2451-2457.

7. H. Song, D. L. Chen and R. F. Ismagilov, Angew. Chem. Int. Edit., 2006, 45, 7336-7356.

8. D. Huh, J. H. Bahng, Y. B. Ling, H. H. Wei, O. D. Kripfgans, J. B. Fowlkes, J. B. Grotberg and S. Takayama, Anal. Chem., 2007, 79, 1369-1376.

9. K. Ahn, C. Kerbage, T. P. Hunt, R. M. Westervelt, D. R. Link and D. A. Weitz, Appl. Phys. Lett., 2006, 88, 024104.

10. N. Pamme and A. Manz, Anal. Chem., 2004, 76, 7250-7256.

11. M. P. MacDonald, G. C. Spalding and K. Dholakia, Nature, 2003, 426, 421-424.

12. J. J. Shi, X. L. Mao, D. Ahmed, A. Colletti and T. J. Huang, Lab Chip, 2008, 8, 221-223.

13. S. Lecuyer, W. D. Ristenpart, O. Vincent and H. A. Stone, Appl. Phys. Lett., 2008, 92 , 104105.

14. D. Di Carlo, D. Irimia, R. G. Tompkins and M. Toner, P. Natl. Acad. Sci. USA, 2007, 104, 18892-18897.

15. D. Di Carlo, Lab Chip, 2009, 9, 3038-3046.

16. J. C. Baret, O. J. Miller, V. Taly, M. Ryckelynck, A. El-Harrak, L. Frenz, C. Rick, M. L. Samuels, J. B. Hutchison, J. J. Agresti, D. R. Link, D. A. Weitz and A. D. Griffiths, Lab Chip, 2009, 9, 1850-1858.

17. C. A. Stan, G. F. Schneider, S. S. Shevkoplyas, M. Hashimoto, M. Ibanescu, B. J. Wiley and G. M. Whitesides, Lab Chip, 2009, 9, 2293-2305.

18. D. Di Carlo, J. F. Edd, K. J. Humphry, H. A. Stone and M. Toner, Phys. Rev. Lett., 2009, 102, 094503.

19. G. Segre and A. Silberberg, Nature, 1961, 189, 209-\&.

20. P. G. Saffman, J. Fluid Mech., 1965, 22, 385-400.

21. H. L. Goldsmith and S. G. Mason, J. Colloid Sci., 1962, 17, 448-476. 
22. P. C. H. Chan and L. G. Leal, J. Fluid Mech., 1979, 92, 131-170.

23. M. Fukuta, S. Takagi and Y. Matsumoto, Phys. Fluids, 2008, 20, 040704.

24. S. Takagi and Y. Matsumoto, Annu. Rev. Fluid Mech., 2011, 43, 615-636.

25. P. J. A. Janssen and P. D. Anderson, Chem. Eng. Res. Des., 2008, 86, 1388-1396.

26. J. A. Hanna and P. M. Vlahovska, Phys. Fluids, 2010, 22, 013102.

27. V. Narsimhan and E. S. G. Shaqfeh, Phys. Fluids, 2010, 22, 101702.

28. J. T. Schwalbe, F. R. Phelan, P. M. Vlahovska and S. D. Hudson, Soft Matter, 2011, 7, 7797-7804.

29. P. S. Williams, T. Koch and J. C. Giddings, Chem. Eng. Commun., 1992, 111, 121-147.

30. K. Sugiyama and F. Takemura, J. Fluid Mech., 2010, 662, 209-231.

31. F. Takemura, J. Magnaudet and P. Dimitrakopoulos, J. Fluid Mech., 2009, 634, 463-486.

32. V. G. Levich, Physicochemical Hydrodynamics, Prentice-Hall, Inc., Englewood Cliffs, NJ, 1962.

33. C. A. Stan, L. Guglielmini, A. K. Ellerbee, D. Caviezel, H. A. Stone and G. M. Whitesides, Phys. Rev. E, 2011, 84, 036302.

34. G. I. Taylor, Proc. Roy. Soc. Lond. A, 1934, 146, 0501-0523.

35. C. A. Stan, S. K. Y. Tang and G. M. Whitesides, Anal. Chem., 2009, 81, 2399-2402.

36. J. P. Matas, J. F. Morris and E. Guazzelli, J. Fluid Mech., 2004, 515, 171-195.

37. T. M. Geislinger, B. Eggart, S. B. Ller, L. Schmid and T. Franke, Appl. Phys. Lett., 2012, 100, 183701.

38. S. C. Hur, N. K. Henderson-MacLennan, E. R. B. McCabe and D. Di Carlo, Lab Chip, 2011, 11, 912-920.

39. S. Mortazavi and G. Tryggvason, J. Fluid Mech., 2000, 411, 325-350. 